\title{
Minimally Invasive Percutaneous Nephrolithotomy versus Retrograde Intrarenal Surgery for Upper Urinary Stones: A Systematic Review and Meta-Analysis
}

\author{
Hongyang Jiang, Zhe Yu, Liping Chen, Tao Wang, Zhuo Liu, \\ Jihong Liu, Shaogang Wang, and Zhangqun Ye \\ Department of Urology, Tongji Hospital, Tongji Medical College, Huazhong University of Science and Technology, Hubei 430030, China \\ Correspondence should be addressed to Zhuo Liu; tjmulz@163.com and Jihong Liu; jhliu@tjh.tjmu.edu.cn
}

Received 12 January 2017; Accepted 5 April 2017; Published 3 May 2017

Academic Editor: Christian Schwentner

Copyright (C) 2017 Hongyang Jiang et al. This is an open access article distributed under the Creative Commons Attribution License, which permits unrestricted use, distribution, and reproduction in any medium, provided the original work is properly cited.

\begin{abstract}
Minimally invasive percutaneous nephrolithotomy (mini-PCNL) and retrograde intrarenal surgery (RIRS) are both alternatives for PCNL to treat renal calculi. This study is aimed at comparing the stone-free rate (SFR) and other surgery parameters of two approaches for treating upper urinary calculi. We performed this meta-analysis in September 2016 by searching studies about miniPCNL and RIRS for treating upper urinary calculi in various databases, and RevMan v.5.3 was applied. Three randomized controlled trials and ten nonrandomized trials were included, involving a total of 1317 patients. Meta-analysis showed that mini-PCNL group led to a higher SFR [odds ratio: 1.96; 95\% confidence interval: 1.46-2.64; $P<0.00001$ ] but brought a larger postoperative decrease in hemoglobin levels compared with RIRS. RIRS provided a shorter hospital time. There was no significant difference in operation time. Higher postoperative complications were detected in the mini-PCNL, but the difference was not significant. Grade I and III complications did not vary between two procedures, but grade II complications were of lower incidence in RIRS group. In the light of these results, compared with RIRS, mini-PCNL provided significantly higher SFR and efficiency quotient for managing calculi; however, it resulted in higher incidence of postoperative complications, larger hemoglobin drops, and longer hospital stay.
\end{abstract}

\section{Introduction}

Kidney calculi is a common urological disorder which is characterized by high recurrence rate [1]. The stone movement leading to renal colic and the obstruction by calculi could result in kidney function loss. Recently, the incidence of kidney calculi has been on the rise in China, probably caused by the changed climate and environment. For releasing the obstruction, urologists choose different treatments for different size calculi diameter from less than $0.6 \mathrm{~cm}$ to more than $3.0 \mathrm{~cm}$. As the guidelines recommend, percutaneous nephrolithotomy (PCNL), of which standard access tracts are 24-30 French $(\mathrm{Fr})$, is a recommended management of patients with renal or ureteral stones more than $20 \mathrm{~mm}$ or and for smaller stones $(10-20 \mathrm{~mm}$ ) of the lower pole stones when anatomic factors make extracorporeal shockwave lithotripsy (ESWL) unfavorable. Although PCNL is suggested as a standard method for its excellent stone-free rate, there is still few surgical drawbacks that may compromise its efficacy [1].
For reducing postoperative morbidity associated with large devices such as blood loss, fever, and potential renal damage, minimally invasive tract has been applied widely. Minimally invasive PCNL (also termed mini-PCNL or miniPerc or mPCNL), a miniature endoscope via a small percutaneous tract $(11-20 \mathrm{Fr})$, is widely executed in the recent years $[2,3]$. Mini-PCNL was described by Helal et al. Firstly performed on a 2-year-old child by the use of instruments with smaller access diameters in 1997 and developed by Jackman et al. to be a therapy option for adults $[4,5]$ compared to the standard tract PCNL, mini-PCNL has a more gracile tract of $<20 \mathrm{Fr}$, which leads to less nephron loss and other postoperative complications; meanwhile the stonefree rate seems to have no significant difference $[6,7]$.

On the other hand, retrograde intrarenal surgery (RIRS) (also termed flexible ureterorenoscopy, F-URS), is another major minimally invasive measure for managing the upper urinary calculi. For its characteristics of little trauma, quick 
recovery, easy operation, and little contraindication, RIRS has been considered as an alternative for the percutaneous approaches for lower pole stones treatment [8,9]. RIRS is a safe procedure with lower complication rates, blood loss, shorter length of stay, and lower stone-free rate than PCNL [10].

Mini-PCNL and RIRS are two effective minimally invasive approaches to release the obstruction. For the question of which one should be the better choice to replace the standard tract PCNL, there is not yet enough high-quality data to provide evidence. Therefore, we conducted this systematic review and meta-analysis of available literatures comparing SFR and other surgery-related parameters of mini-PCNL to RIRS for the treatment of kidney calculi.

\section{Materials and Methods}

2.1. Studies Selection. This meta-analysis was performed in September 2016 using PubMed, Cochrane Library, Embase, and Web of Science databases to identify related studies in accordance with the meta-analysis (PRISMA) guidelines (http://www.prismastatement.org) and preferred reporting items for a systematic review. Search strategy was as follows: (kidney stone OR urolithiasis OR kidney calculus OR kidney calculi OR renal stone OR nephrolith OR renal calculus) AND (mini-PCNL OR mPCNL OR minimally invasive surgery OR minimally invasive percutaneous nephrolithotomy OR minipercutaneous OR miniaturized PCNL OR ultra-mini-PCNL) AND (retrograde intrarenal surgery OR RIRS OR flexible ureteroscopy OR flexible ureterorenoscopy OR retrograde ureterolithotripsy).

Before the study search, we circumscribed inclusion criteria including (1) patients with kidney calculi, (2) the age $>18$, (3) comparing mini-PCNL with RIRS, (4) reporting at least one of the following outcomes (operative time, SFR, hemoglobin drop, hospitalization time, or postoperative complications), and (5) related parameters that could be obtained from the studies. And exclusion criteria were as follows: (1) nephrostomy tract size in mini-PCNL $>20 \mathrm{~F}$ or $<11 \mathrm{~F}$; (2) conference abstracts which were not deemed to be methodologically appropriate; (3) non-English papers; (4) the inclusion criteria that were not met. Two authors accomplished the review process independently. A third author arbitrated disagreements in data extraction by consensus.

2.2. Data Quality Assessment. As shown in Table 1, we rated the level of evidence (LE) of every included trail according to the Oxford Centre for Evidence-Based Medicine Criteria [24]. The qualities of nonrandomized controlled trials (nonRCTs) were assessed according to Newcastle-Ottawa Scale (NOS), and RCTs qualities were assessed by the Jadad scale $[25,26]$. Two reviewers performed the procedure independently and all disagreements were resolved by consensus.

2.3. Data Extraction and Statistical Analysis. All metaanalyses were performed to assess the overall outcomes using Review Manager Software (RevMan v.5.3, Cochrane Collaboration, Oxford, UK). Extracted data for the analysis included stone-free rate, operative time, hemoglobin drop, length of hospital stay, and postoperative complication rate. If standard deviations were not reported we estimated data according to Hozo SP [27], and if standard deviation (SD) could not be calculated from available data, we asked authors to supply the data. For evaluating dichotomous variables, we chose odds ratio (OR), and, for continuous parameters, weighted mean difference (WMD) or standardized mean difference was used. The Cochrane chi-square test and inconsistency $\left(I^{2}\right)$ were used to evaluate the heterogeneity among studies. Pooled estimates were calculated with the fixed-effect model for stone-free rate, and the other outcomes were calculated with the random-effect model due to the heterogeneity. Funnel plots were generated using RevMan v.5.3 to evaluate published bias of literatures. In addition, we included highquality studies (RCTs and high score non-RCTs (NOS: 7 of 9 points)) into meta-analysis for sensitive analysis.

\section{Results}

3.1. Study Characteristics and Quality. Thirteen trails were selected for analysis including a total of $701 \mathrm{mini}-\mathrm{PCNL}$ cases (53.2\%) and 616 RIRS cases (46.8\%) in our meta-analysis. The literature screening process is shown in Figure 1.

The baseline characteristics of the included studies are shown in Tables 2 and 3. Surgical technique for mini-PCNL differed in aspect of tract size and lithotripsy. As shown in Table 1, the studies consisted of three RCTs (LE: 2b) [11, 16, 22], two matched-pair analysis trails (LE: 3b) [13, 20], and eight case control studies (LE: $3 \mathrm{~b}$ ) including 4 prospective $[14,17,21,23]$ and 4 retrospective studies $[12,15,18,19]$. In terms of the methodological quality, eight and two of the nonrandomized studies were relatively high (NOS: 7 of 9 points and 6 of 9 points) and medium (NOS: 5 of 9 points and 4 of 9 points), respectively. The three RCTs were of medium quality (Jadad scale: 3 of 5 points). In 3 studies, mini-PCNL and RIRS were compared between patients with a single stone. There were also two studies comparing mini-PCNL with RIRS for proximal ureteral stones, whereas the rest were compared for renal calculi. In general, the preoperative demographic characteristics such as mean age (51.80 \pm 14.80 versus $49.9 \pm 14.29$ year) were comparable between miniPCNL and RIRS.

\section{The Results of Parameters in Meta-Analysis}

4.1. Stone-Free Rate (SFR). SFR was evaluated with fixedeffect model, and the comparison of stone-free rate between the mini-PCNL and RIRS group is shown in Figure 2. All involved studies reported postoperative stone-free rate, and the result suggested that mini-PCNL group provided a significantly higher SFR than RIRS group (OR: 1.96; 95\% CI, $1.46-2.64 ; P<0.00001)$ with no significant homogeneity $\left(I^{2}=42 \%\right)$. As the stone location subgroup meta-analysis shows (Figure 3), mini-PCNL has significantly higher SFR than RIRS in any location (OR: 2.13 ; 95\% CI, 1.53-2.96; $P<$ $0.00001)$. Figure 4 shows that mini-PCNL can lead to higher stone clearance in both 1-2 cm (OR: 2.01; 95\% CI, 1.27-3.19; 


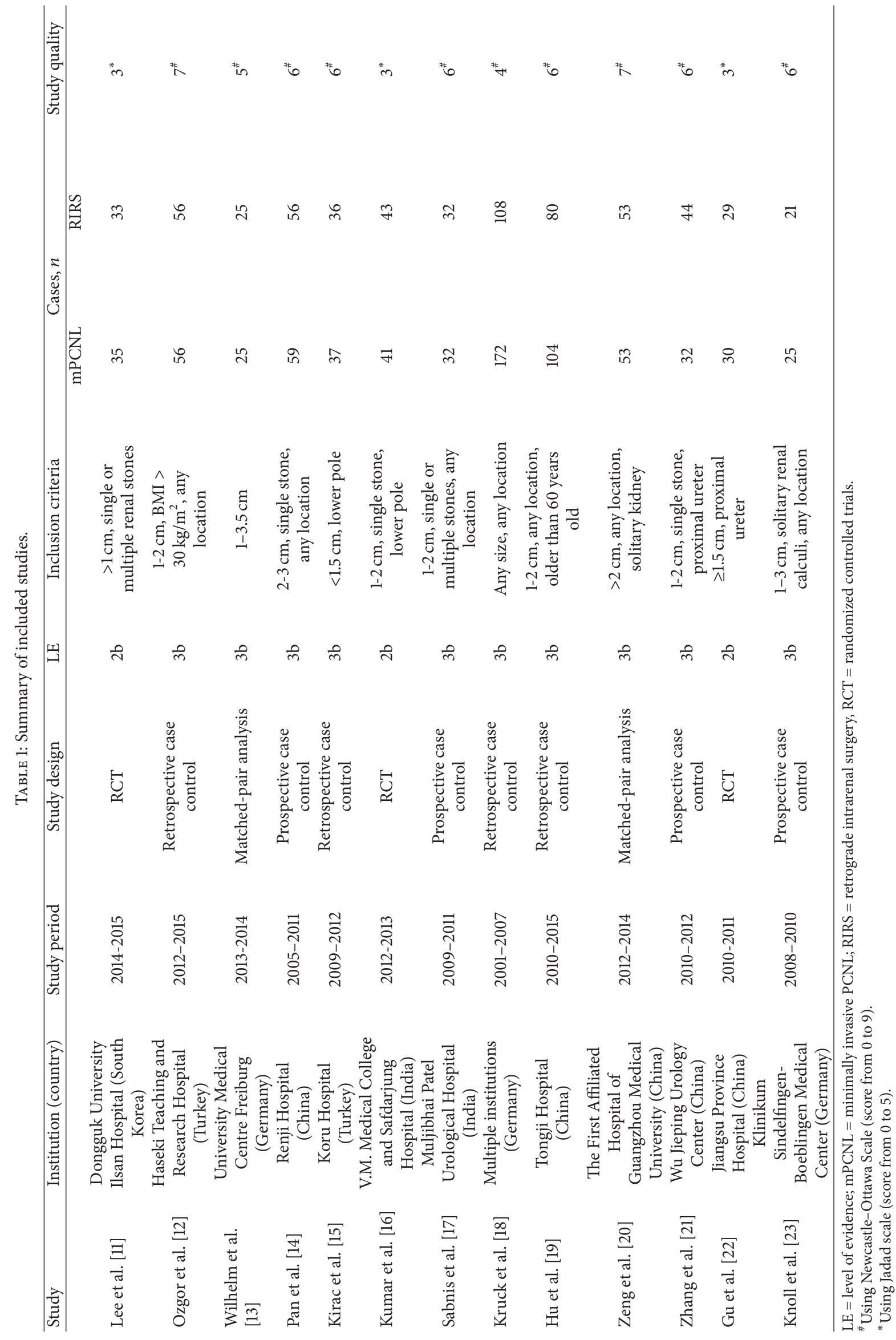




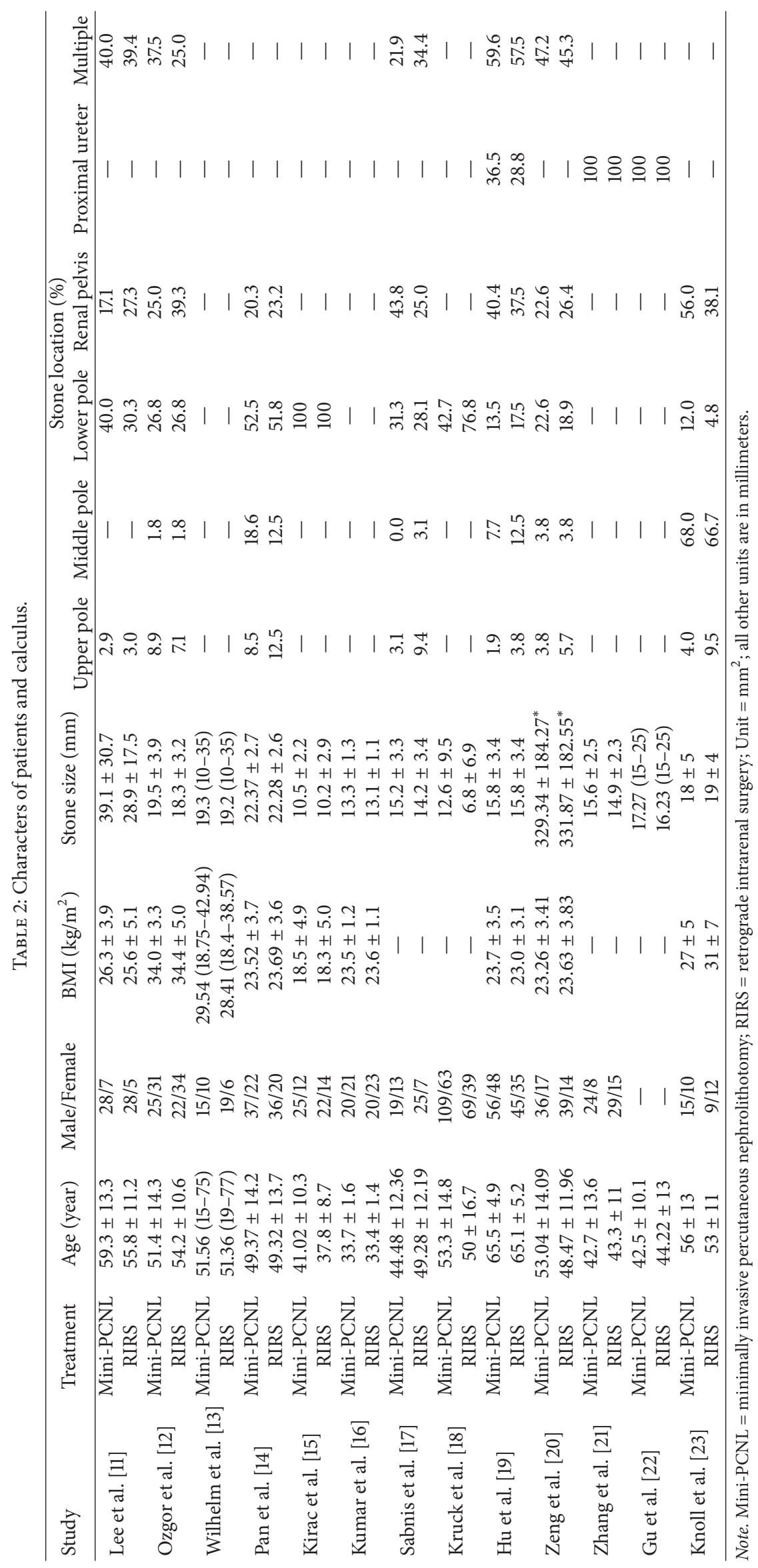


TABLE 3: The characters of the surgical methods of included studies.

\begin{tabular}{|c|c|c|c|c|c|}
\hline Study & Treatment & Access sheath size, Fr & Dilator & Nephroscope size & Lithotripsy \\
\hline \multirow{2}{*}{ Lee et al. [11] } & Mini-PCNL & 18 & Balloon & $15 \mathrm{~F}$ & Laser \\
\hline & RIRS & $14 / 16$ & UAS & $7.5 \mathrm{~F}$ & Laser \\
\hline \multirow{2}{*}{ Ozgor et al. [12] } & Mini-PCNL & 18 or 20 & Amplatz & $17 \mathrm{~F}$ & Laser and ultrasound \\
\hline & RIRS & $19 / 23$ & UAS & $7.5 \mathrm{~F}$ & Laser \\
\hline \multirow{2}{*}{ Wilhelm et al. [13] } & Mini-PCNL & 10 and 14 & PTFE dilators/Amplatz & $13 \mathrm{~F}$ & Laser \\
\hline & RIRS & $7 / 8$ & UAS & - & Laser \\
\hline \multirow{2}{*}{ Pan et al. [14] } & Mini-PCNL & 18 & Amplatz & $14 \mathrm{~F}$ & Laser \\
\hline & RIRS & 12 & UAS & Olympus P3 or P5 & Laser \\
\hline \multirow{2}{*}{ Kirac et al. [15] } & Mini-PCNL & 20 & Amplatz & $15-16.5 \mathrm{~F}$ & Pneumatic or ultrasound energy \\
\hline & RIRS & $9.5 / 11.5$ or $12 / 14$ & UAS & 8 or $9.5 \mathrm{~F}$ & Laser \\
\hline \multirow{2}{*}{ Kumar et al. [16] } & Mini-PCNL & 18 & gauge needle & $15 \mathrm{~F}$ & Pneumatic \\
\hline & RIRS & 12 & UAS & $8 / 9.8 \mathrm{~F}$ & Laser \\
\hline \multirow{2}{*}{ Sabnis et al. [17] } & Mini-PCNL & $16-19$ & 22-gauge Skinny Needle & $15 / 18 \mathrm{~F}$ and $16.5 / 19.5 \mathrm{~F}$ & Laser \\
\hline & RIRS & 14 & UAS & 7.5-F Flex X-2 & Laser \\
\hline \multirow{2}{*}{ Kruck et al. [18] } & Mini-PCNL & $16-18$ & Metal & $12 \mathrm{~F}$ & Ultrasound \\
\hline & RIRS & - & Fascial dilator & Flex-X/Flex-X2 & Laser \\
\hline \multirow{2}{*}{ Hu et al. [19] } & mPCNL & $16-20$ & Fascial dilator & $8 / 9.8 \mathrm{~F}$ & Laser \\
\hline & RIRS & $12 / 14$ & UAS & Flex-X2 & Laser \\
\hline \multirow{2}{*}{ Zeng et al. [20] } & Mini-PCNL & 18 & Fascial dilators & - & Laser and pneumatic \\
\hline & RIRS & $12 / 14$ & UAS & $7.5 \mathrm{~F}$ & Laser \\
\hline \multirow{2}{*}{ Zhang et al. [21] } & Mini-PCNL & $18-20$ & facial dilators & $8.6 / 9.8 \mathrm{~F}$ & Laser and pneumatic \\
\hline & RIRS & $12 / 14$ & UAS & $5.3-8.4 \mathrm{~F}$ & Laser \\
\hline \multirow{2}{*}{ Gu et al. [22] } & Mini-PCNL & $12 / 18$ & Fascial dilators & $8.5 / 9.8 \mathrm{~F}$ & Laser \\
\hline & RIRS & - & UAS & $7.4 \mathrm{~F}$ & Laser \\
\hline \multirow{2}{*}{ Knoll et al. [23] } & Mini-PCNL & 18 & Amplatz & $14 \mathrm{~F}$ & Laser \\
\hline & RIRS & $12 / 14$ & - & - & Laser \\
\hline
\end{tabular}

Mini-PCNL = minimally invasive percutaneous nephrolithotomy; RIRS = retrograde intrarenal surgery; UAS = ureteral access sheath placement.

$P=0.003$ ) and $>2 \mathrm{~cm}$ subgroups (OR: 2.65 ; 95\% CI, $1.81-3.87$; $P<0.0001)$.

4.2. Operative Time. Twelve studies combined had reported operative time, which was evaluated with random effect model. As the meta-analysis result shows in Figure 5(a), there was no remarkable difference between mini-PCNL and RIRS (WMD, $-2.21 ; 95 \% \mathrm{CI},-12.62-8.20 ; P=0.68)$.

4.3. Hospital Stay. In 10 studies there were available data to extract in terms of hospital stay, which was analyzed by random effect model. As shown in Figure 5(b), hospital stay for RIRS is shorter than mini-PCNL (WMD: $1.63 \mathrm{~d}$; $95 \% \mathrm{CI}$, $0.98-2.28 ; P<0.00001)$.

4.4. Hemoglobin ( $\mathrm{Hb}$ ) Drop. Hb drop was analyzed by random effect model, and the result was shown in Figure 5(c). Six studies provided accessible data about $\mathrm{Hb}$ drop and the metaanalysis shows that RIRS led to less Hb drop than mini-PCNL (WMD, 0.60; 95\% CI, 0.32-0.88; $P<0.0001$ ).

4.5. Complication Rate. All studies reported available data for the assessment of the complications between mini-PCNL and RIRS group. Random effect model and OR were used for statistical analysis and the result is shown in Figure 6. RIRS has a lower complication rate than mini-PCNL (OR: 1.62 ; 95\% CI, 0.92-2.88; $P=0.10)$. Furthermore, we analyzed the complications according to Clavien-Dindo Classification (Table 4) to evaluate minor indisposition and major complication $[28,29]$. As the results show, there is no significant difference between grade I and grade III complications between the two groups (OR: 1.24, 95\% CI, 0.66-2.32, $P=0.51$; OR: 1.41, 95\% CI, 0.97-2.04, $P=0.77$ ); however, we observed a significantly lower incidence of grade II complications in RIRS group (OR: 1.63; 95\% CI, 1.01-2.63; $P=0.04$ ) (Figure 7).

4.6. Sensitivity Analysis. The sensitivity analysis suggested that the results of this meta-analysis were relatively stable (Table 5). When only RCTs and high score non-RCTs (NOS: 7 of 9 points) were included, most of the outcomes including stone-free rate, operative time, total postoperative complications, and grade I and III surgery complications were not greatly changed. Meanwhile, significant differences of grade II complications, hemoglobin drop, and hospital stay between two groups were not found because of the reduced sample capacity. It is notable that even if the significant differences 


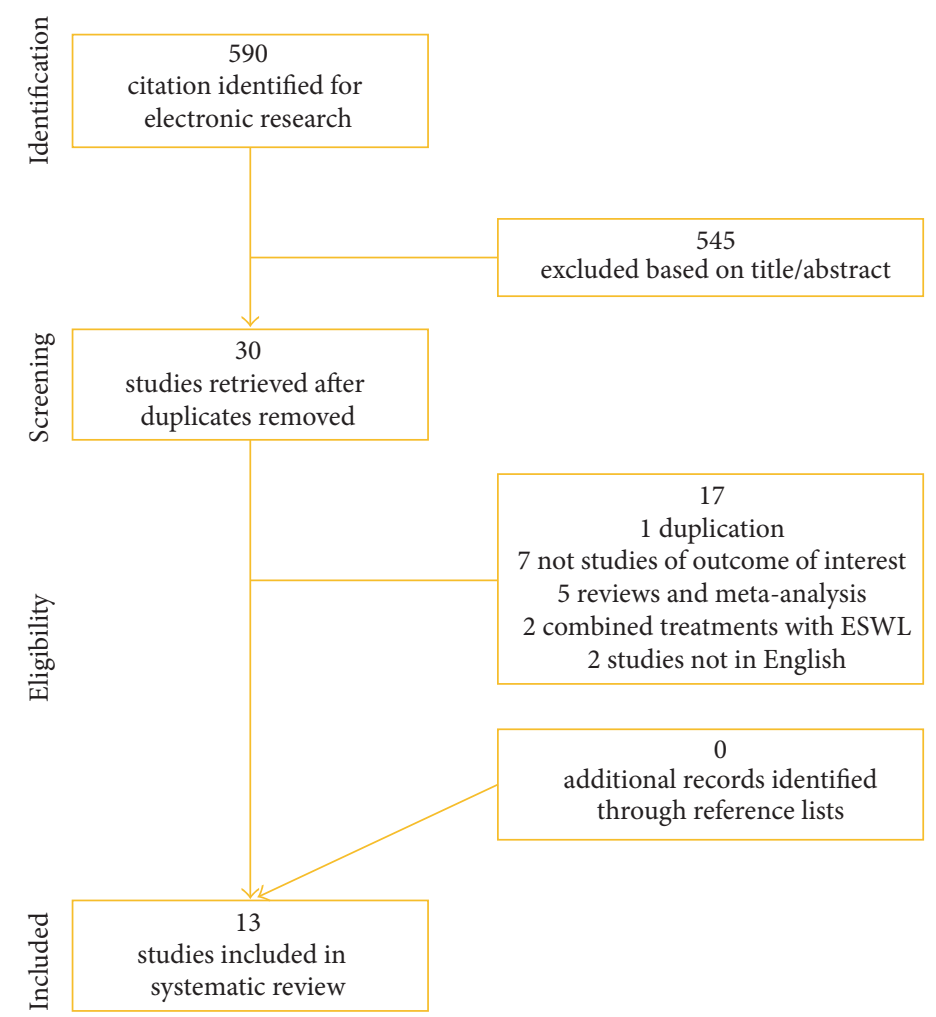

FIgURE 1: Study flow chart.

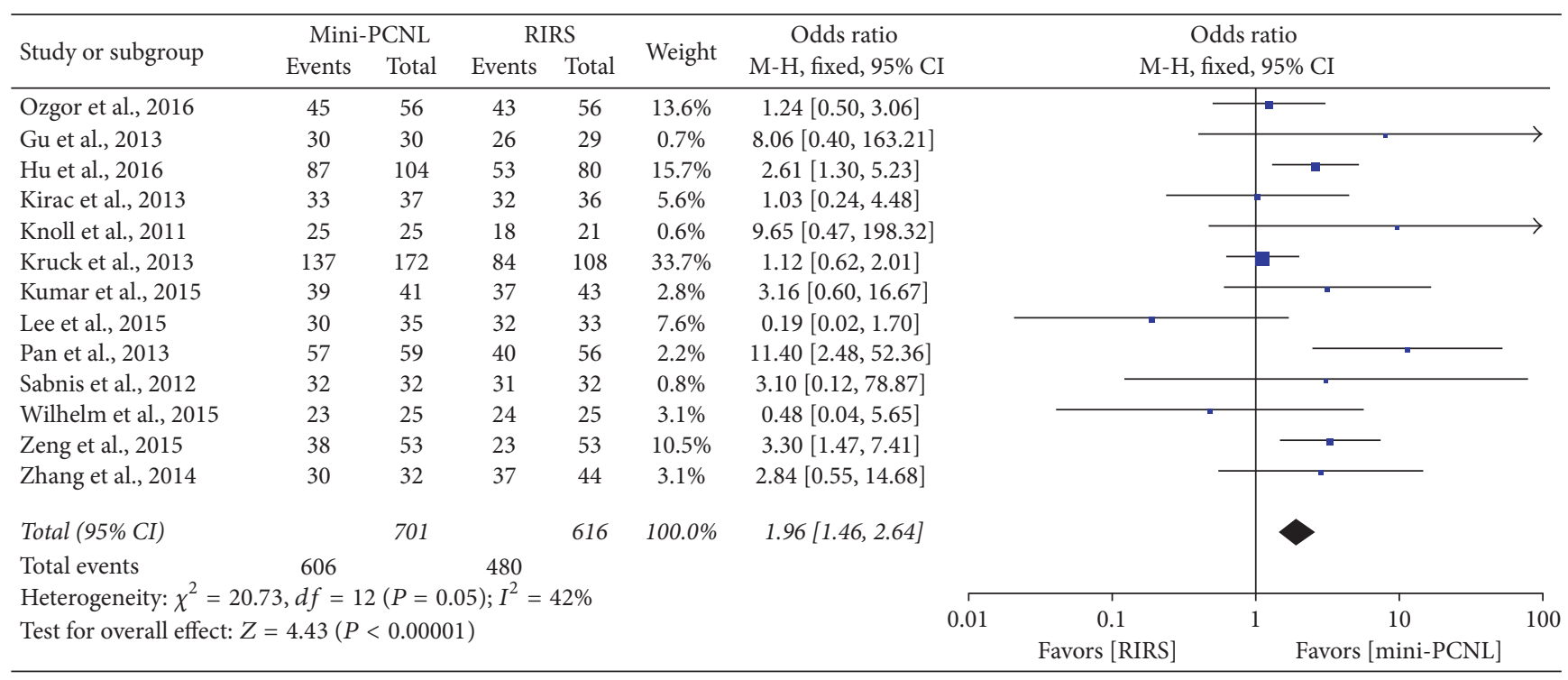

FIGURE 2: Forest plot and meta-analysis of stone-free rate in renal stone patients.

were no longer detectable in the sensitivity analysis, the tendency of meta-analysis stayed in the same direction.

4.7. Publish Bias Analysis. The funnel plot (Figure 8) showed an apparent asymmetry, which suggested the existence of a potential publication bias.

\section{Discussion}

With high technological advancement, urologists who take charge of urolithiasis are in possession of high technique instruments, which leads to safer and more effective lithotripsy. So far PCNL is considered to be the recommended therapy for large stones $>2.0 \mathrm{~cm}$ by both AUA and EAU 


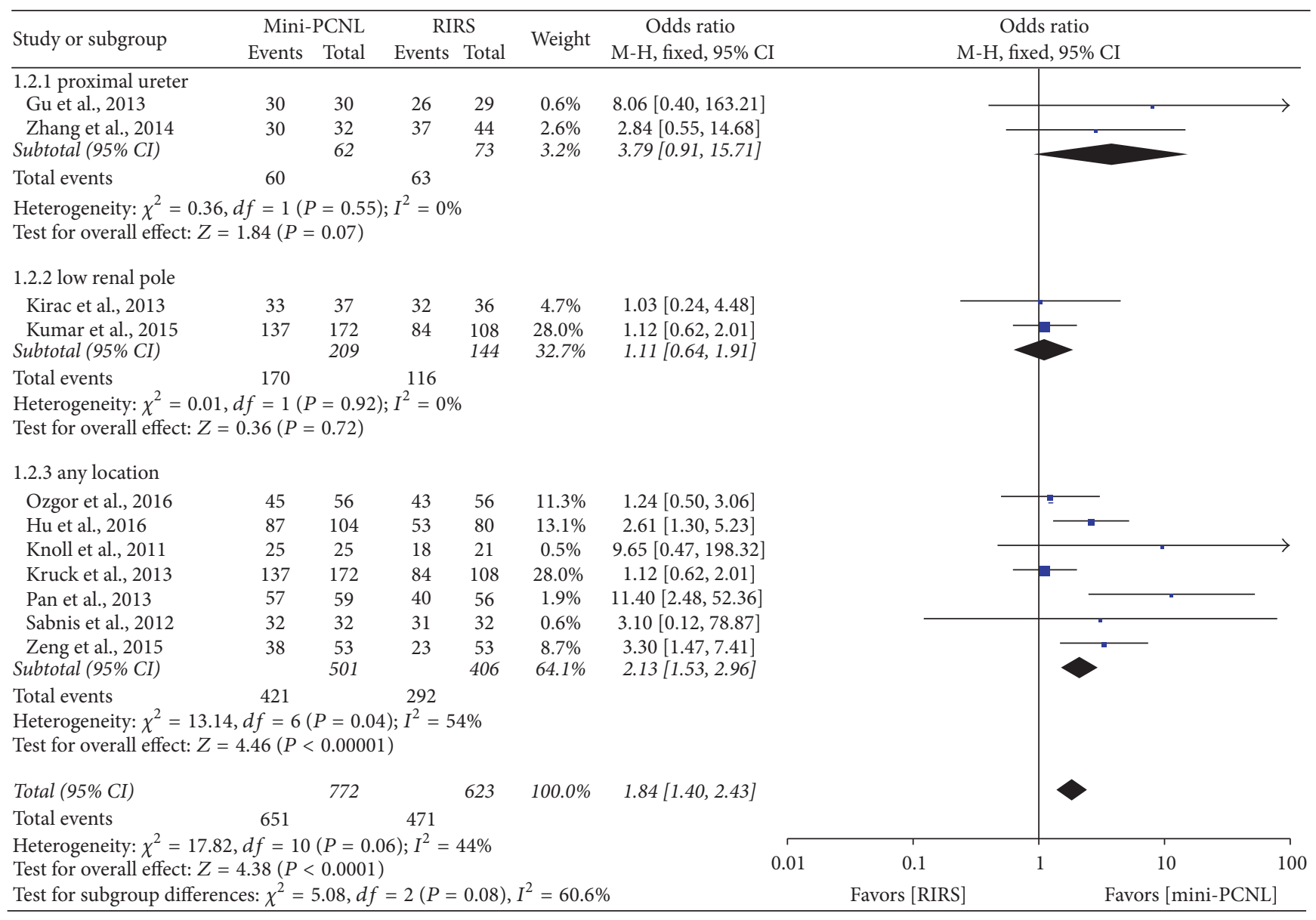

FIGURE 3: Forest plot and meta-analysis of location subgroup of stone-free rate.

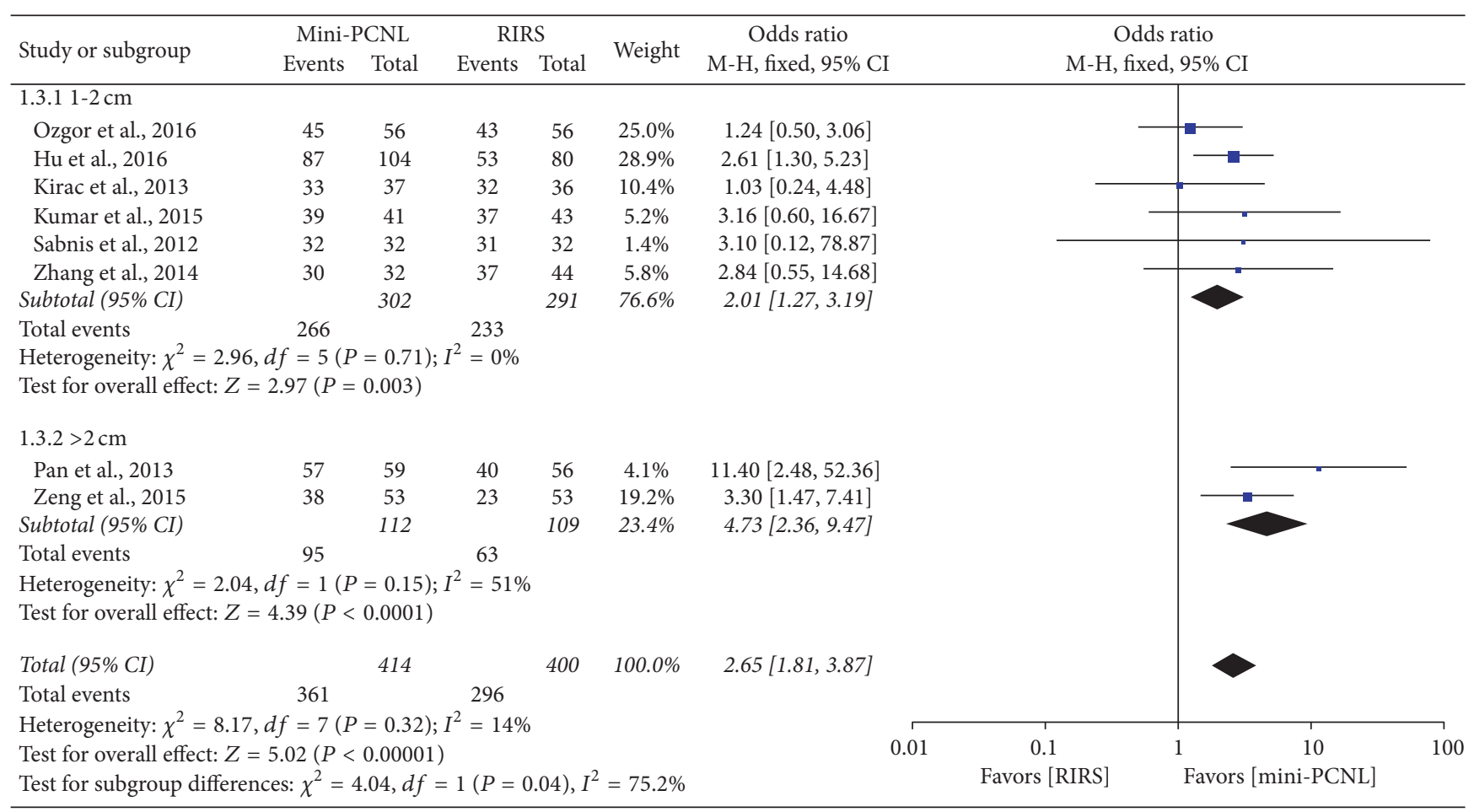

FIGURE 4: Forest plot and meta-analysis of stone size subgroup of stone-free rate. 


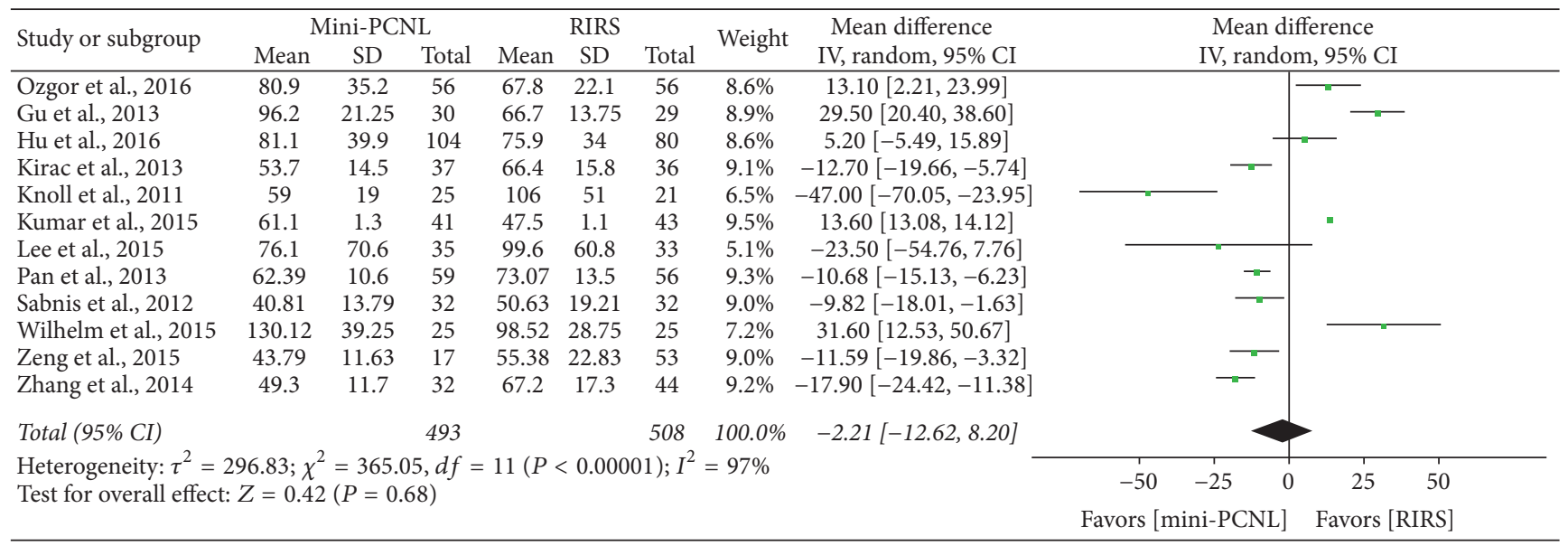

(a)

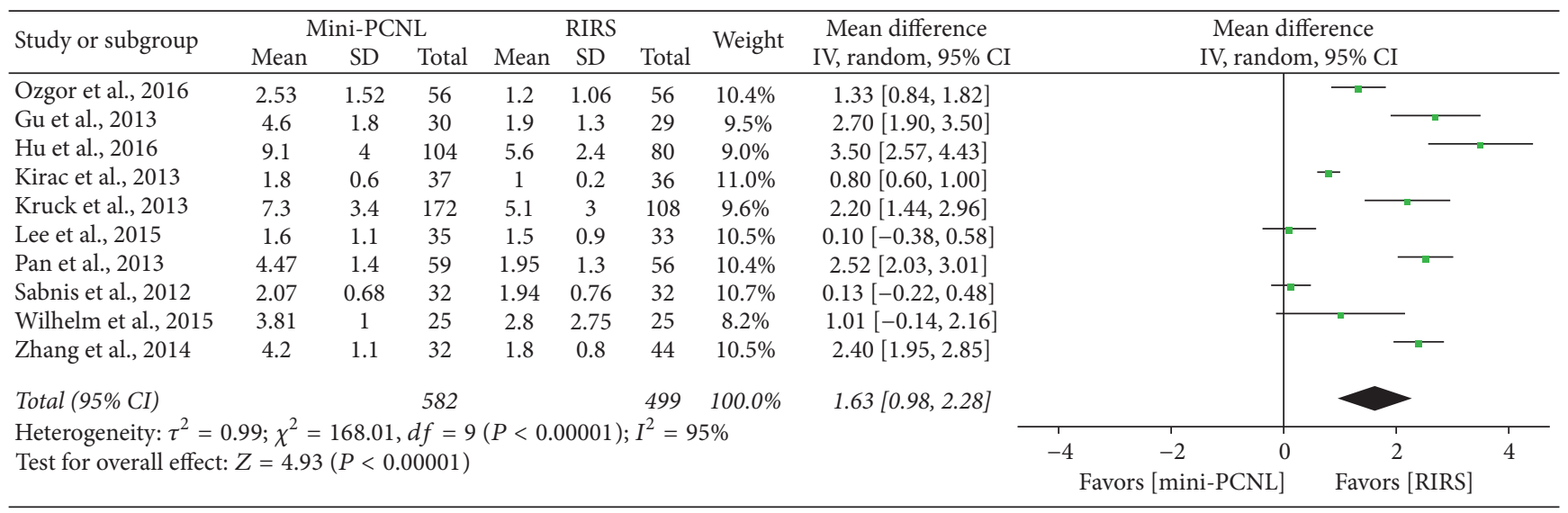

(b)

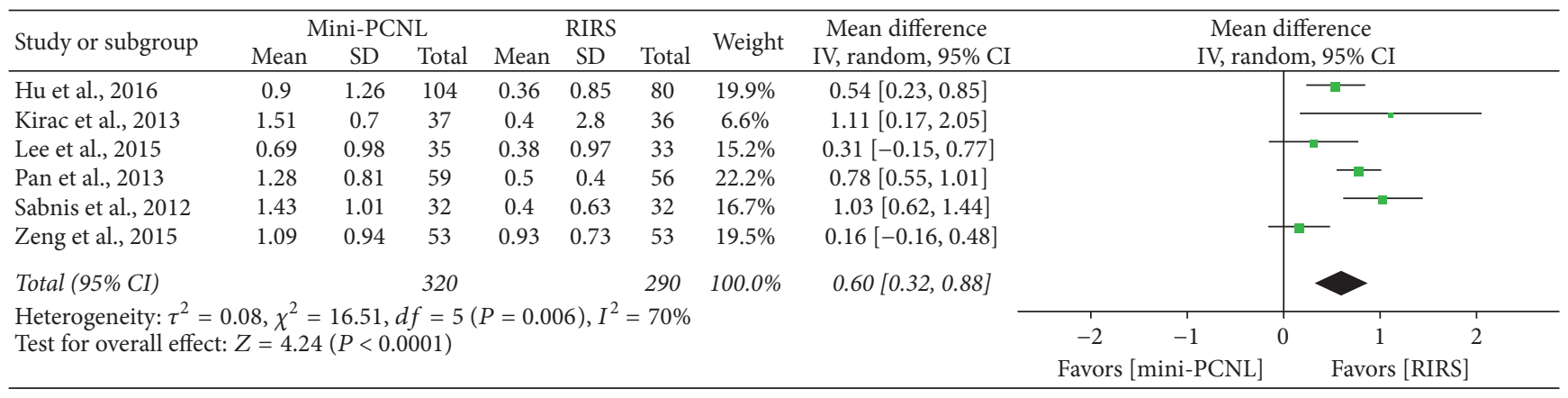

(c)

FIGURE 5: Forest plot and meta-analysis of outcomes in renal stone patients: (a) operative time; (b) hospital stay; (c) hemoglobin drop.

guidelines. Furthermore, with the development of the "miniPCNL" procedure, smaller access sheaths $(\leq 20 \mathrm{~F})$ are becoming increasingly popular for its relative safety. Besides, recent reports suggested that RIRS is a safer approach that could lead to less complications and $\mathrm{Hb}$ drop than normal tract PCNL. We conducted this meta-analysis to systematically analyze the outcomes of two miniature procedures, mini-PCNL and RIRS, which cause considerably lesser trauma than standard PCNL, to find which one could lead to better efficacy and safety. And, to the best of our knowledge, this meta-analysis is an update analysis comparing these two modern minimally invasive approaches applying for upper urinary stone.

SFR is the most important parameter for estimating the efficacy of two approaches. According to the synthesis analysis of data, mini-PCNL has a higher SFR than RIRS group though there were various imaging modalities to identify. Stone-free rates are correlated with the lithotripsy and the location or size of stones. Seven inclusive studies used only laser to dispose stones and others made use of pneumatic or ultrasound waves to fragment calculi. 
TABLE 4: Clavien-Dindo Classification for surgical complication.

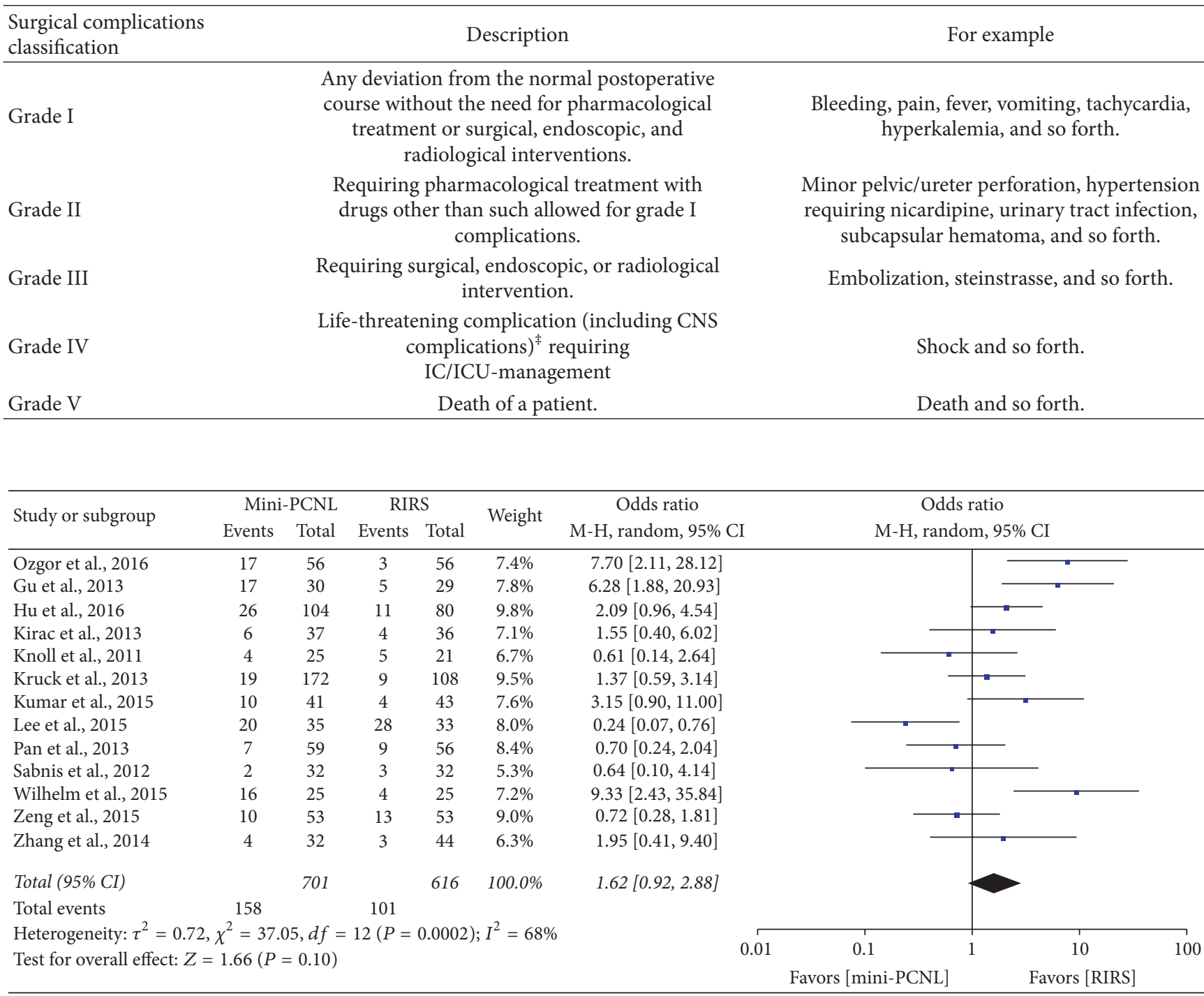

FIGURE 6: Forest plot and meta-analysis of total complications for two procedures.

Zhang et al. and $\mathrm{Gu}$ et al. included only proximal ureter stones and almost all included trails studied stones $>10 \mathrm{~mm}$. To evaluate the different locations that may impact SFR of two procedures, a subgroup analysis was performed. As Figure 3 shows, the proximal and low pole subgroups did not show any remarkable advantage of two approaches, while all locations showed that mini-PCNL has a significant advantage in SFR. Additionally, one stone size subgroup analysis was performed to estimate the impact on meta-analysis; results showed that mini-PCNL has more efficiency stone clearance in both $1-2 \mathrm{~cm}$ and $>2 \mathrm{~cm}$ groups (Figure 4 ). Besides, miniPCNL carries high efficiency quotient (EQ) (Table 6), which was related to SFR, percentage retreatment, and percentage requiring an auxiliary procedure (as the following formula) $[30,31]$, reported by three included studies (Table 5) [14, 16, 22]. However, De et al. had performed a meta-analysis that compared PCNL and RIRS for managing kidney stone and the results showed that RIRS can provide higher stone-free rates compared with $\mathrm{mPCNL}$, which was opposite to our results [10]. It should be noted that only 5 literatures were included in the previous study, and the "mPCNL" in this study referred to micronephroscope which is $4.85 \mathrm{Fr}$ and mini-PCNL from 11 to 19 Fr. This diversity of definition and sample size may result in the outcomes' difference between our meta-analysis and the previous study. Including more relative studies, the outcome would become more reliable.

$$
\mathrm{EQ}^{31}=\frac{\text { precentage of stone-free }}{100+\text { precentage of retreatment }+ \text { precentage of auxiliary procedure }} .
$$




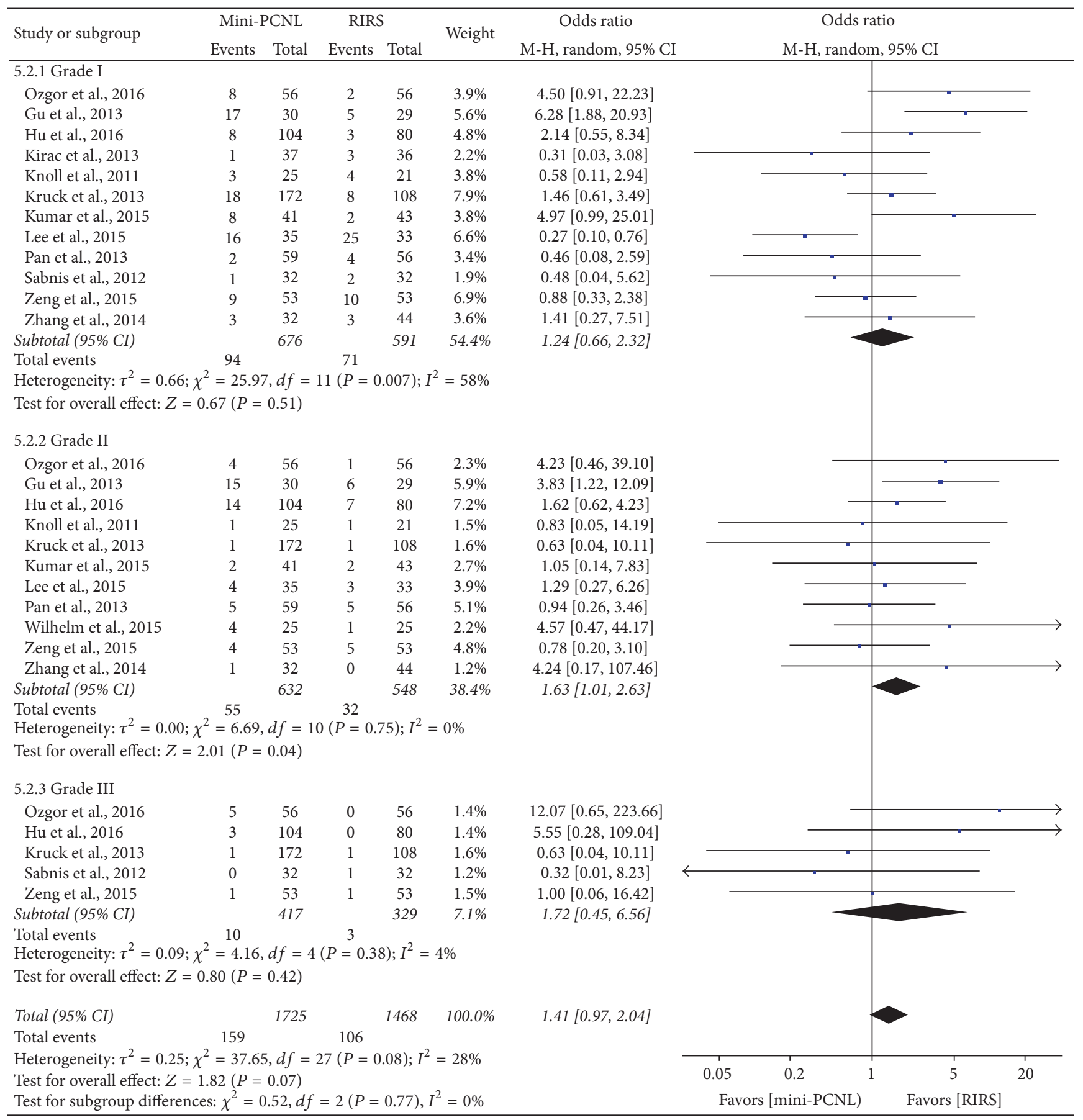

FIGURE 7: Forest plot and meta-analyses of postoperative complications.

Operative times were reported in 12 involved studies, and six studies indicated that mini-PCNL spent shorter operating time, while four studies favored RIRS. The overall metaanalysis showed that two procedures brought no significantly varied operation time; meanwhile, we noticed the heterogeneity in this section was as high as $97 \%$, mainly led by Kumar et al., Gu et al., Ozgor et al., and Wilhelm et al. [12, $13,16,22]$. If the four studies were excluded, heterogeneities would be declined to $70 \%$, and the preference would favor mini-PCNL procedure. Operative time is closely related to nuance in surgical techniques and doctors' experience, different surgeons in different centers provided a large variation in operative time, and a significant heterogeneity was proved from twelve inclusive studies.

The overall analysis found that RIRS resulted in shorter hospital stay than mini-PCNL group. The reason for this 


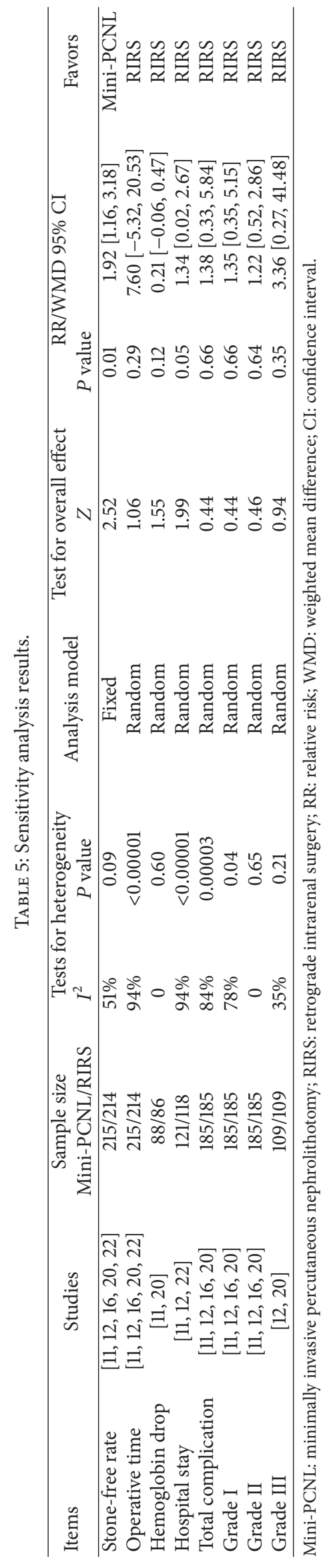


TABLE 6: Efficiency quotient in included studies.

\begin{tabular}{lccc}
\hline Study & EQ for mini-PCNL & EQ for RIRS & $P$ value \\
\hline Pan et al. [14] & 0.904 & 0.523 & - \\
Kumar et al. [16] & 0.915 & 0.842 & 0.01 \\
Gu et al. [22] & 0.830 & 0.500 & - \\
\hline
\end{tabular}

$\mathrm{EQ}=$ efficiency quotient.

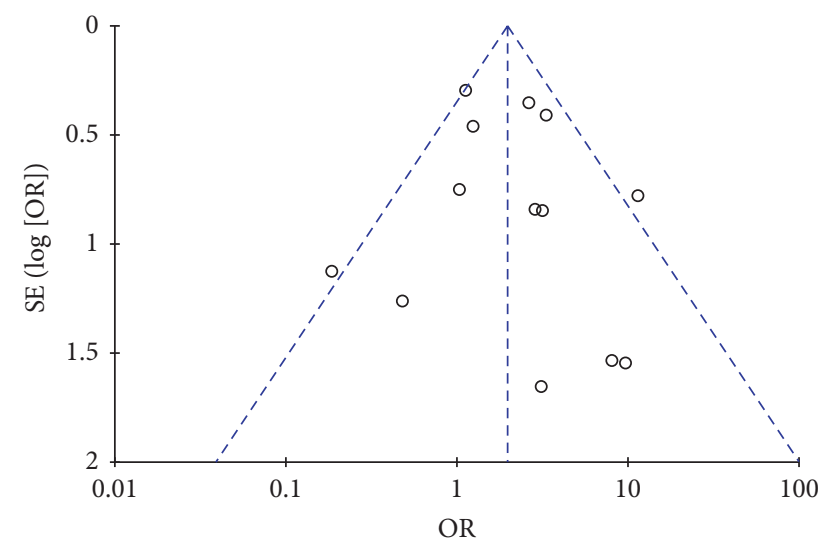

FIGURE 8: Funnel plot for the publication bias test of mini-PCNL versus RIRS.

difference might be less invasive caused by RIRS. Moreover, it carries lower complication rate and hemoglobin drop.

The size of the tract is one key factor for blood loss during endourology surgery, so mini-PCNL with miniature tract can reduce bleeding and the risk of blood transfusion compared to normal tract PCNL [32]. Besides, the overall analysis of the literature suggested that RIRS resulted in less hemoglobin drop than mini-PCNL. Accordingly, RIRS has a high efficiency for the management of intrarenal stones with a slight complication to patients $[33,34]$.

All trails have made the comparison of postoperative morbidity between mini-PCNL and RIRS. The results showed that RIRS provided a lower complication rate than miniPCNL; however, the difference had no significance. The complications of mini-PCNL are similar to those of PCNL; bleeding, pain, and fever are very common [35-38].

Furthermore, we performed a subgroup meta-analysis of postoperative complications, classifying them into grades I, II, and III based on Clavien-Dindo Classification, between the two groups [28]. As Table 4 shows, grade I represents the morbidities that needed no pharmacological or surgical treatment, which could easily occur after operation, and grade III means complications requiring surgical, endoscopic, or radiological intervention, which rarely occur after lithotripsy operation. Thus, we did not observe a remarkable difference in comparison of grade I. Grade III complications were only observed in 5 studies, and the result showed that RIRS has a potential safety on severe complications. As for grade II complications, mini-PCNL has a significantly higher rate than RIRS according to our meta-analysis, which means RIRS was probably safer with respect to middle or severe morbidities after operation, and, in term of light deviations, the incidences of mini-PCNL and RIRS were similar.

There are several limitations in the present meta-analysis. In our systematic review and meta-analysis, we included the currently available comparative studies. Although we have done the sensitivity analysis to show that the results were relatively stable, there is still some bias of our conclusion caused by non-RCTs. Besides, heterogeneities among involved literatures, which may relate to diverse calculi size and location, different tract size, and lithotripsies, could lead to some limitations in our meta-analysis. In addition, most of the included trials failed to describe complications with the same criteria and blinding procedures in detail, and this might lead to conclusion bias, as the more details the literatures describe, the more credible the conclusion will be concluded. However, to the best of our knowledge, this study is one update review and meta-analysis to compare mini-PCNL and RIRS for treating renal calculi. We believe the results of the present meta-analysis could help urologists make better clinical decisions to manage stone disease patients.

\section{Conclusions}

From this meta-analysis, we found that both mini-PCNL and RIRS can provide safe and effective treatment for renal calculi patients. In the light of these results, compared with RIRS, mini-PCNL provided significantly higher stonefree rate and efficiency quotient for management of upper urinary calculi, however, could increase the incidence of postoperative complications and the average hospital stay.

\section{Disclosure}

This article does not contain any studies with humans or animals performed by any of the authors. The funders had no role in study design, data collection and analysis, decision to publish, or preparation of the manuscript.

\section{Conflicts of Interest}

All authors declare no conflicts of interest.

\section{Authors' Contributions}

Hongyang Jiang, Zhuo Liu, and Tao Wang conceived and designed the experiments. Hongyang Jiang, Zhe Yu, and Liping Chen performed the experiments. Hongyang Jiang, Zhe Yu, and Liping Chen analyzed the data. Jihong Liu, Zhangqun Ye, and Shaogang Wang contributed 
reagents/materials/analysis tools. Hongyang Jiang, Zhe Yu, and Liping Chen wrote the paper.

\section{Acknowledgments}

This work was supported by the National Natural Science Foundation of China (Grant no. 81400706).

\section{References}

[1] D. Prezioso, M. Di Martino, R. Galasso, and G. Iapicca, "Laboratory assessment," Urologia Internationalis, vol. 79, supplement 1, pp. 20-25, 2007.

[2] N. Ferakis and M. Stavropoulos, "Mini percutaneous nephrolithotomy in the treatment of renal and upper ureteral stones: lessons learned from a review of the literature," Urology Annals, vol. 7, no. 2, pp. 141-148, 2015.

[3] J. Rassweiler, M.-C. Rassweiler, and J. Klein, "New technology in ureteroscopy and percutaneous nephrolithotomy," Current Opinion in Urology, vol. 26, no. 1, pp. 95-106, 2016.

[4] M. Helal, T. Black, J. Lockhart, and T. E. Figueroa, "The Hickman peel-away sheath: alternative for pediatric percutaneous nephrolithotomy," Journal of Endourology, vol. 11, no. 3, pp. 171$172,1997$.

[5] S. V. Jackman, S. G. Docimo, J. A. Cadeddu, J. T. Bishoff, L. R. Kavoussi, and T. W. Jarrett, "The 'mini-perc' technique: a less invasive alternative to percutaneous nephrolithotomy," World Journal of Urology, vol. 16, no. 6, pp. 371-374, 1998.

[6] W. Zhu, Y. Liu, L. Liu et al., "Minimally invasive versus standard percutaneous nephrolithotomy: a meta-analysis," Urolithiasis, vol. 43, no. 6, pp. 563-570, 2015.

[7] S. C. Druskin and J. B. Ziemba, "Minimally invasive ('Mini') percutaneous nephrolithotomy: classification, indications, and outcomes," Current Urology Reports, vol. 17, no. 4, article 30, 2016.

[8] T. Akman, M. Binbay, F. Ozgor et al., "Comparison of percutaneous nephrolithotomy and retrograde flexible nephrolithotripsy for the management of 2-4 cm stones: a matched-pair analysis," BJU International, vol. 109, no. 9, pp. 1384-1389, 2012.

[9] E. S. Hyams, R. Munver, V. G. Bird, J. Uberoi, and O. Shah, "Flexible ureterorenoscopy and holmium laser lithotripsy for the management of renal stone burdens that measure 2 to $3 \mathrm{~cm}$ : a multi-institutional experience," Journal of Endourology, vol. 24, no. 10, pp. 1583-1588, 2010.

[10] S. De, R. Autorino, F. J. Kim et al., "Corrigendum re: 'percutaneous nephrolithotomy versus retrograde intrarenal surgery: a systematic review and meta-analysis' [Eur Urol 2015;67: 125-37]," European Urology, vol. 69, p. e85, 2016.

[11] J. W. O. Lee, J. Park, S. B. A. Lee, H. Son, S. Y. O. Cho, and H. Jeong, "Mini-percutaneous nephrolithotomy vs retrograde intrarenal surgery for renal stones larger than $10 \mathrm{~mm}$ : a prospective randomized controlled trial," Urology, vol. 86, no. 5, pp. 873-877, 2015.

[12] F. Ozgor, A. Tepeler, F. Elbir et al., "Comparison of miniaturized percutaneous nephrolithotomy and flexible ureterorenoscopy for the management of $10-20 \mathrm{~mm}$ renal stones in obese patients," World Journal of Urology, vol. 34, no. 8, pp. 1169-1173, 2016.

[13] K. Wilhelm, S. Hein, F. Adams, D. Schlager, A. Miernik, and M. Schoenthaler, "Ultra-mini PCNL versus flexible ureteroscopy: a matched analysis of analgesic consumption and treatmentrelated patient satisfaction in patients with renal stones 10-35 mm," World Journal of Urology, vol. 33, no. 12, pp. 2131-2136, 2015.

[14] J. Pan, Q. Chen, W. Xue et al., "RIRS versus mPCNL for single renal stone of $2-3 \mathrm{~cm}$ : clinical outcome and cost-effective analysis in Chinese medical setting," Urolithiasis, vol. 41, no. 1, pp. 73-78, 2013.

[15] M. Kirac, Ö. F. Bozkurt, L. Tunc, C. Guneri, A. Unsal, and H. Biri, "Comparison of retrograde intrarenal surgery and minipercutaneous nephrolithotomy in management of lower-pole renal stones with a diameter of smaller than $15 \mathrm{~mm}$," Urolithiasis, vol. 41, no. 3, pp. 241-246, 2013.

[16] A. Kumar, N. Kumar, P. Vasudeva, S. Kumar Jha, R. Kumar, and H. Singh, "A prospective, randomized comparison of shock wave lithotripsy, retrograde intrarenal surgery and miniperc for treatment of 1 to $2 \mathrm{~cm}$ radiolucent lower calyceal renal calculi: a single center experience," Journal of Urology, vol. 193, pp. 160164, 2015.

[17] R. B. Sabnis, J. Jagtap, S. Mishra, and M. Desai, "Treating renal calculi $1-2 \mathrm{~cm}$ in diameter with minipercutaneous or retrograde intrarenal surgery: a prospective comparative study," BJU International, vol. 110, no. 8, pp. E346-E349, 2012.

[18] S. Kruck, A. G. Anastasiadis, T. R. W. Herrmann et al., "Minimally invasive percutaneous nephrolithotomy: an alternative to retrograde intrarenal surgery and shockwave lithotripsy," World Journal of Urology, vol. 31, no. 6, pp. 1555-1561, 2013.

[19] H. Hu, Y. Lu, D. He et al., "Comparison of minimally invasive percutaneous nephrolithotomy and flexible ureteroscopy for the treatment of intermediate proximal ureteral and renal stones in the elderly," Urolithiasis, vol. 44, no. 5, pp. 427-434, 2016.

[20] G. Zeng, W. Zhu, J. Li et al., "The comparison of minimally invasive percutaneous nephrolithotomy and retrograde intrarenal surgery for stones larger than $2 \mathrm{~cm}$ in patients with a solitary kidney: a matched-pair analysis," World Journal of Urology, vol. 33, no. 8, pp. 1159-1164, 2015.

[21] Y. Zhang, C.-F. Yu, S.-H. Jin, H. Zhu, and Y.-Q. Na, "A prospective comparative study between minimally invasive percutaneous nephrolithotomy in supine position and flexible ureteroscopy in the management of single large stone in the proximal ureter," Urology, vol. 83, no. 5, pp. 999-1002, 2014.

[22] X. J. Gu, J. L. Lu, and Y. Xu, "Treatment of large impacted proximal ureteral stones: randomized comparison of minimally invasive percutaneous antegrade ureterolithotripsy versus retrograde ureterolithotripsy," World Journal of Urology, vol. 31, no. 6, pp. 1605-1610, 2013.

[23] T. Knoll, J. P. Jessen, P. Honeck, and G. Wendt-Nordahl, "Flexible ureterorenoscopy versus miniaturized PNL for solitary renal calculi of 10-30 mm size," World Journal of Urology, vol. 29, no. 6, pp. 755-759, 2011.

[24] B. Phillips, C. Ball, D. Sackett, D. Badenoch, and S. Straus, "Oxford centre for evidence-based medicine levels of evidence," Verfügbar unter, http://www.cebm.net/oxford-centreevidence-based-medicine-levels-evidence-march-2009/.

[25] H. D. Clark, G. A. Wells, C. Huët et al., "Assessing the quality of randomized trials: reliability of the Jadad scale," Controlled Clinical Trials, vol. 20, no. 5, pp. 448-452, 1999.

[26] G. A. Wells, B. Shea, D. O'connell et al., “The Newcastle-Ottawa Scale (NOS) for assessing the quality of nonrandomised studies in meta-analyses," 2000. 
[27] S. P. Hozo, B. Djulbegovic, and I. Hozo, "Estimating the mean and variance from the median, range, and the size of a sample," BMC Medical Research Methodology, vol. 5, article 13, 2005.

[28] D. Dindo, N. Demartines, and P.-A. Clavien, "Classification of surgical complications: a new proposal with evaluation in a cohort of 6336 patients and results of a survey," Annals of Surgery, vol. 240, no. 2, pp. 205-213, 2004.

[29] M. Balc1, A. Tuncel, Ö. Güzel et al., "Evaluation of the complications in transperitoneal laparoscopic renal and adrenal surgery with Clavien-Dindo classification," Turk Uroloji Dergisi, vol. 42, no. 2, pp. 70-73, 2016.

[30] G. M. Preminger, H.-G. Tiselius, D. G. Assimos et al., "2007 Guideline for the management of ureteral calculi," European Urology, vol. 52, no. 6, pp. 1610-1631, 2007.

[31] H. K. Salem, "A prospective randomized study comparing shock wave lithotripsy and semirigid ureteroscopy for the management of proximal ureteral calculi," Urology, vol. 74, no. 6, pp. 1216-1221, 2009.

[32] H. Sun, Z. Zhang, J. Yuan et al., "Safety and efficacy of minimally invasive percutaneous nephrolithotomy in the treatment of patients with medullary sponge kidney," Urolithiasis, vol. 44, no. 5, pp. 421-426, 2016.

[33] D. J. Galvin and M. S. Pearle, "The contemporary management of renal and ureteric calculi," BJU International, vol. 98, no. 6, pp. 1283-1288, 2006.

[34] O. M. Aboumarzouk, M. Monga, S. G. Kata, O. Traxer, and B. K. Somani, "Flexible ureteroscopy and laser lithotripsy for stones $>2 \mathrm{~cm}$ : a systematic review and meta-analysis," Journal of Endourology, vol. 26, no. 10, pp. 1257-1263, 2012.

[35] R. Goel, M. Aron, P. K. Kesarwani, P. N. Dogra, A. K. Hemal, and N. P. Gupta, "Percutaneous antegrade removal of impacted upper-ureteral calculi: still the treatment of choice in developing countries," Journal of Endourology, vol. 19, no. 1, pp. 54-57, 2005.

[36] L.-Y. Li, X. Gao, M. Yang et al., "Does a smaller tract in percutaneous nephrolithotomy contribute to less invasiveness? A prospective comparative study," Urology, vol. 75, no. 1, pp. 5661, 2010.

[37] L. Quaresima, A. Conti, A. Vici et al., "Safety and efficacy of percutaneous nephrolithotomy in the Galdakao-modified Supine Valdivia position: a prospective analysis," Archivio Italiano di Urologia e Andrologia, vol. 88, no. 2, pp. 93-96, 2016.

[38] W. Zhu, J. Li, J. Yuan et al., "A prospective and randomised trial comparing fluoroscopic, total ultrasonographic, and combined guidance for renal access in mini-percutaneous nephrolithotomy," BJU International, vol. 119, no. 4, pp. 612-618, 2017. 


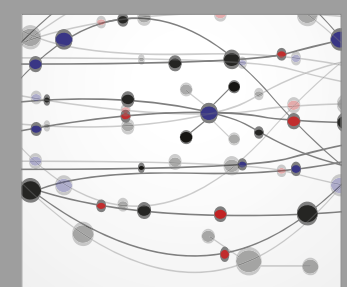

The Scientific World Journal
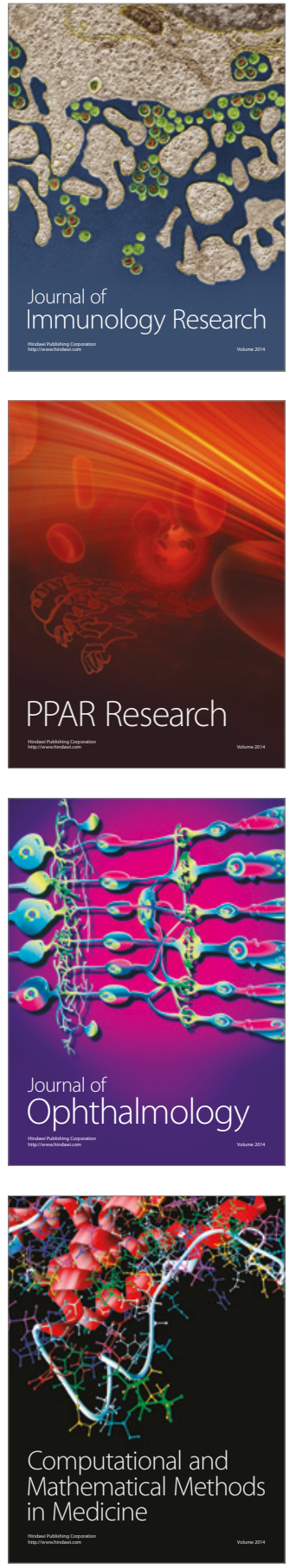

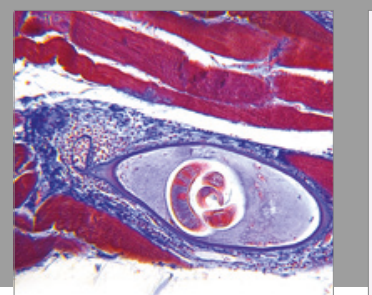

Gastroenterology Research and Practice
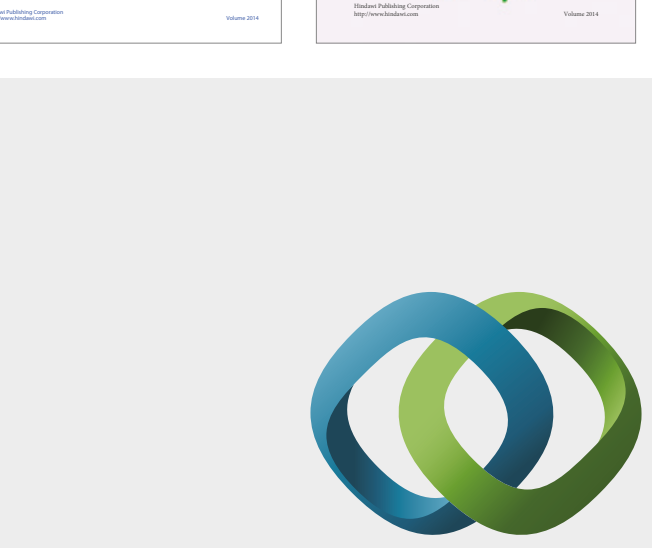

\section{Hindawi}

Submit your manuscripts at

https://www.hindawi.com
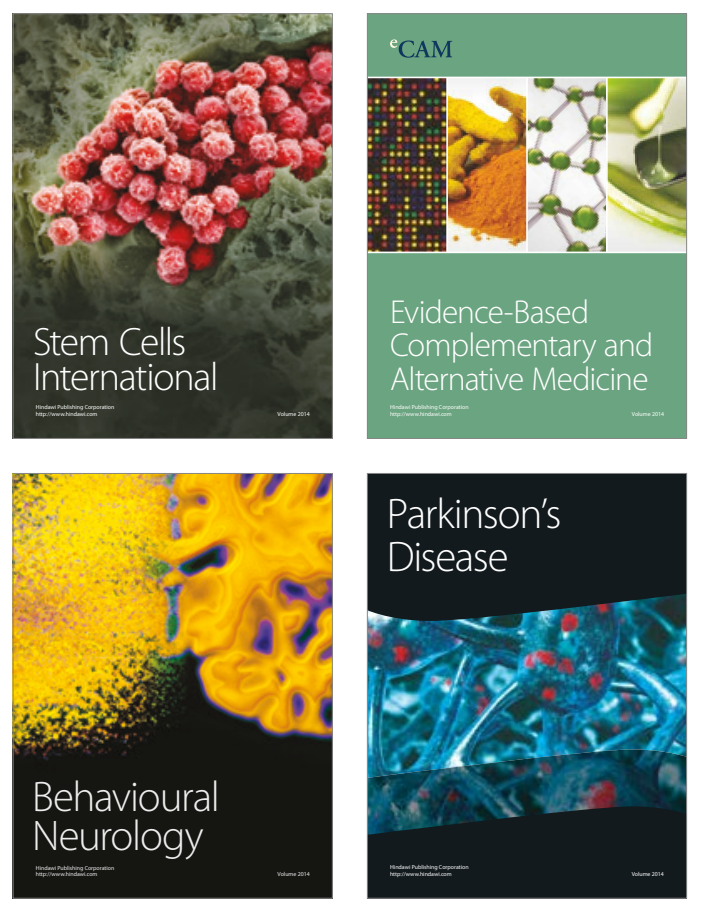
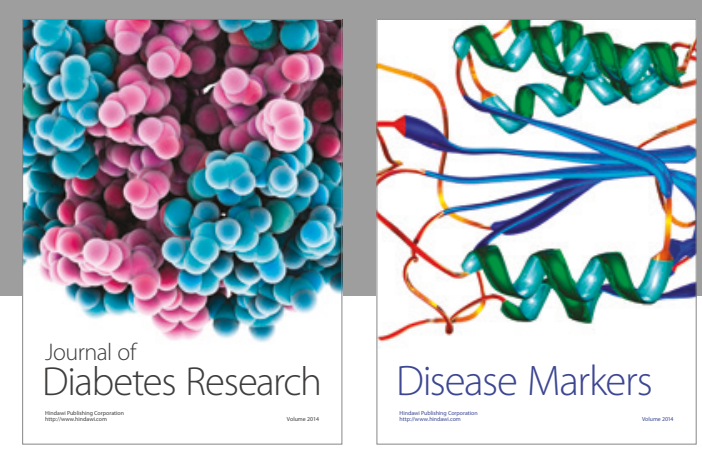

Disease Markers
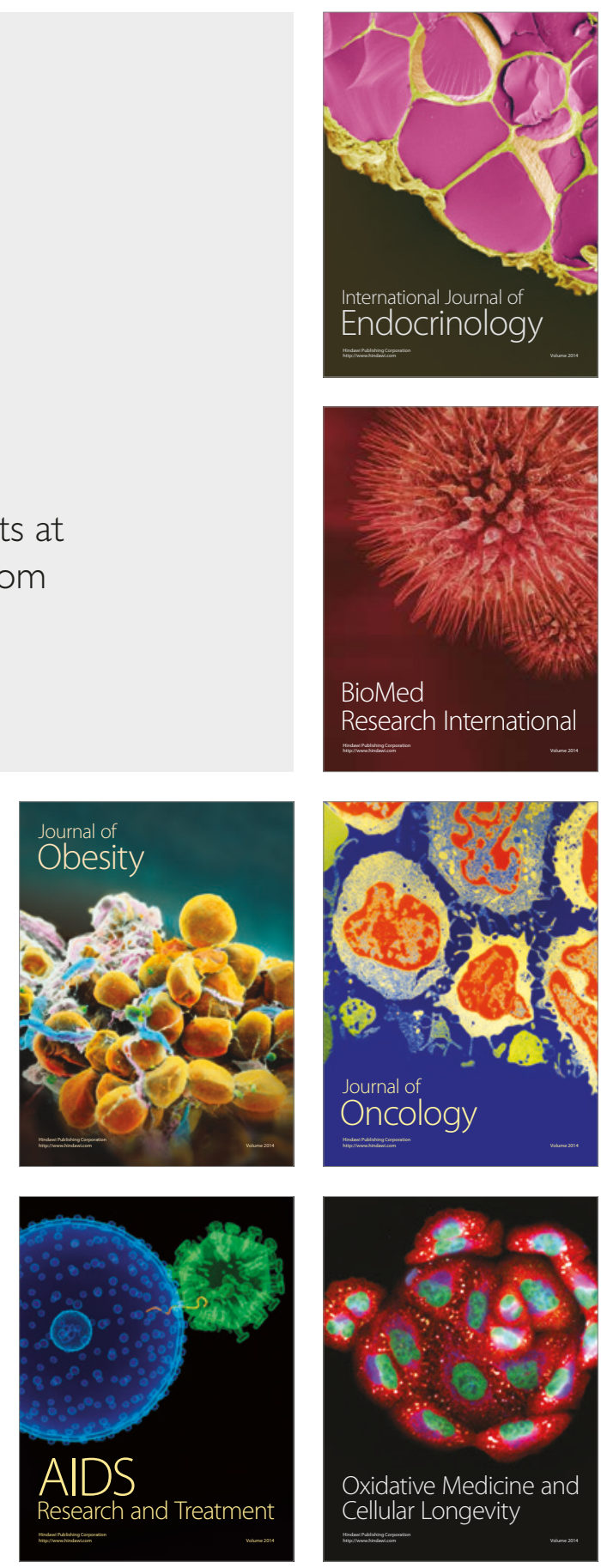